Advances in Radio Science (2003) 1: 43-47

(C) Copernicus $\mathrm{GmbH} 2003$

\title{
Impact of near field dispersion on time domain susceptibility tests
}

\author{
F. Sabath ${ }^{1}$ and H. Garbe ${ }^{2}$ \\ ${ }^{1}$ Wehrwissenschaftliches Institut für Schutztechnologien - ABC-Schutz, Postfach 1142, 29623 Munster, Germany \\ ${ }^{2}$ Institut für Grundlagen der Elektrotechnik und Messtechnik, Universität Hannover, Appelstrasse 9A, 30167 Hannover, \\ Germany
}

\begin{abstract}
This paper considers the effect of changes of the waveform parameters over the distance. The objective is to show the near field effects on transient radiation and how near the equipment under test can be located to an antenna without significant changes of the width or rise time of the field pulse. It is shown that the well known frequency domain estimation models fail because the dimension of antenna systems and the wave propagation lead to a special kind of dispersion. The importance of this near field dispersion for practical applications is demonstrated by both theoretical considerations and measurements with a half impulse radiation antenna.
\end{abstract}

\section{Introduction}

Electronic components and subsystems (e.g. microprocessor boards) are essential parts of modern civilian and military systems like airplanes, communication, traffic management or safety systems. A setup or failure in these systems could cause a major accident or economic disaster. Due to the rising possibility of modern electronic systems to be threatened by fast transient electromagnetic fields of high intensity their susceptibility is of great interest.

For susceptibility investigations using transient field signals (transient susceptibility tests) a setup consisting of a high power impulse generator, a suitable impulse forming network (PFN) and an impulse radiating antenna is used. The development of new ultra wide band (UWB) sources and antennas has shown significant progress in the recent years. In particular, research in impulse radiating antennas (IRAs) has improved the performance of a number of other wide band systems. The development of a theoretical description of IRAs has been considered for some years. Now theoretical models are available for the far field radiation of various antenna types (e.g. reflector type, TEM-horns, antenna

Correspondence to: F. Sabath

(FrankSabath@bwb.org) arrays). Due to the strong impact of the waveform on the coupling of transient fields into systems, the parameters of the waveform like rise time or pulse duration are often more important than the pulse amplitude for susceptibility investigations. Based on the limited amplitude variability of high power pulse sources the field magnitudes are often scaled by changing the distance between antenna and target or by changing the direction angle. Hereby is the direction angle defined as the angle between the direction of the main beam an the line from the center of the antenna to the location of the EUT in the horizontal plane. The radiated pulse shape depends on the direction angle (e.g. the rise time decreases for increasing direction angle).

In this paper we focus on the effect of distance changes on the waveform parameters (e.g. rise time, pulse duration). The main goal is to show how near the equipment under test (EUT) can be located by the antenna without significant parameter changes of the pulse shape and which effects occur in the intermediate and the near field. The paper begins with a short review of an analytical model for radiation of aperture antennas. Following this, a review of the frequency domain far field definition is performed. Based on these theoretical reviews a time domain far field definition will be developed with respect to waveform effects. The comparison of the developed definition and the classical frequency domain definitions shows that for fast transient radiation the well known frequency domain estimation model fails because the dimension of antenna systems and the wave propagation lead to a special kind of dispersion. The importance of this near field dispersion for practical applications is demonstrated by both theoretical considerations and measurements with a half impulse radiation antenna (HIRA II).

\section{Time domain susceptibility test setup}

Investigations of the effects of fast transient electromagnetic fields on modern electronic systems generally use a measurement setup consisting of a high power pulse source, a pulse- 
forming network and a field generating device. Field pulses with a $10-90 \%$ rise time in the 100 ps regime and a $50 \%$ pulse width of less than $5 \mathrm{~ns}$ can be generated by

- TEM-waveguides (open stripline, GTEM-Cell,...)

- impulse radiating antennas (IRAs)

- Small conical antennas

On the one hand the TEM-waveguides are characterized by a nondispersive transfer function and a field homogeneity of high quality. On the other hand the behavior of higher order modes limit the useable size of the test volume. In contrast to the TEM-waveguides antennas are able to illuminate whole systems, but they are characterized by a derivative transfer function. In general time domain susceptibility measurements are similar to the common measurement procedures in frequency domain. Both procedures start with a calibration of the incident test field in absence of the EUT. Following this, the EUT is located in the center of the defined test volume and is illuminated by the test field. The amplitude of the test field is increased gradually until malfunctions or destruction's are observed. Due to unstabilities of the pulse sources the amplitude of the test field should be monitored by a reference probe during the whole test.

The field amplitude can only be scaled by the use of attenuator or changes of the distance between the antenna and the EUT, because the amplitude range of the output voltage of the available high power pulse sources is restricted or the amplitude variation causes a change of the pulse shape. In case attenuators are used, they must be able to diminish nondispersively voltage pulses with a pulse amplitude of several $100 \mathrm{kV}$ an a bandwidth up to $10 \mathrm{GHz}$. In the standard measurement procedure the amplitude of the test field pulse is varied by changing the distance between the antenna and the EUT, because commercial UWB-attenuators are restricted to pulse amplitudes lower than $15 \mathrm{kV}$. For impulse radiating antennas as well as antenna arrays with an aperture diameter of less than $2 \mathrm{~m}$ far field conditions are assumed for distances larger than $8 \mathrm{~m}$ with regard to $\mathrm{cw}$ measurement procedures. This assumption neglects the influence of transient near field effects on the shape and the spectrum of the field pulse. This article focus on both the applicability of the frequency domain far field assumptions on transient radiation and the influence of transient near field effects on the radiated field pulse.

\section{Radiation of aperture antennas}

The radiation behavior of most impulse radiating antennas (IRA) can be represented by the electrical field $\left(E_{a}\right)$ inside the aperture $\left(A_{a}\right)$ using the equivalence principle (Balanis, 1997). In the frequency domain the electrical field strength at an arbitrary observation point $r_{p}$ can be calculated by the integral

$$
\boldsymbol{E}_{\boldsymbol{s}}\left(\boldsymbol{r}_{\boldsymbol{p}}, s\right)=\frac{1}{2 \pi} \int_{A_{a}} \frac{s R+c}{c R^{2}}
$$

$$
\cdot\left[\left(\boldsymbol{e}_{z} \times \boldsymbol{E}_{\boldsymbol{a}}\left(\boldsymbol{r}_{\boldsymbol{q}}, s\right)\right) \times \boldsymbol{e}_{\boldsymbol{R}}\right] \cdot e^{-\frac{s R}{c}} d A_{a}
$$

with the complex frequency $s=p+j \omega$, the speed of light $c$, the source point $r_{q}$ and the distance

$$
\begin{aligned}
& R=\left|\boldsymbol{r}_{\boldsymbol{p}}-\boldsymbol{r}_{\boldsymbol{q}}\right|= \\
& r_{p}-\frac{2 \cdot \boldsymbol{e}_{\boldsymbol{p}} \cdot \boldsymbol{r}_{\boldsymbol{q}}}{r_{p}+\sqrt{r_{p}^{2} r_{q}^{2}-2 \boldsymbol{e}_{\boldsymbol{p}} \boldsymbol{r}_{\boldsymbol{q}}}}+\frac{r_{q}^{2}}{r_{p}+\sqrt{r_{p}^{2}+r_{q}^{2}-2 \boldsymbol{e}_{\boldsymbol{p}} \boldsymbol{r}_{\boldsymbol{q}}}}
\end{aligned}
$$

between an arbitrary source point inside the aperture an the observing point. The equation describes the radiated field under both near and far field conditions. For a large observation distance $r_{p}$ (far field) the approximations

$$
\begin{aligned}
& R=\sqrt{r_{p}^{2}+r_{q}^{2}-2 \boldsymbol{r}_{\boldsymbol{p}} \boldsymbol{r}_{\boldsymbol{q}}} \approx r_{p}-\boldsymbol{e}_{\boldsymbol{p}} \boldsymbol{r}_{\boldsymbol{q}} \\
& \frac{s R+c}{c R^{2}} \approx \frac{s}{c r_{p}}
\end{aligned}
$$

can be used to simplify Eq. (1):

$$
\begin{aligned}
& \boldsymbol{E}_{\boldsymbol{F a r}}\left(\boldsymbol{r}_{\boldsymbol{p}}, s\right)=\frac{1}{2 \pi c r_{p}} e^{-\frac{s}{c} \cdot r_{p}} . \\
& \cdot \int_{A_{a}}\left[\left(\boldsymbol{e}_{z} \times \boldsymbol{E}_{\boldsymbol{a}}\left(\boldsymbol{r}_{\boldsymbol{q}}, s\right)\right) \times \boldsymbol{e}_{\boldsymbol{p}}\right] \cdot e^{\frac{s}{c} \cdot \boldsymbol{e}_{\boldsymbol{p}} \cdot \boldsymbol{r}} d A_{a}
\end{aligned}
$$

This far field formulation for the radiated electrical field strength consists of two independent terms. The first one describes the wave propagation (phase shift and amplitude attenuation) as a function of the observation distance. The second term, the integral over the aperture area, is a function of the field distribution inside the aperture and the direction angle. In the far field formulation (3) just the phase (or time delay) and the attenuation of the radiated field is a function of the observation distance $r_{p}$. As a result the amplitude of the radiated field can be scaled by changing the distance if the far field approximation is applicable. For normal applications, the recent parameter of interest is the observation distance at which the errors caused by the approximations (3) and (4) are smaller than 5\%, because an error of less than this limit is acceptable.

Following the far field approximations are evaluated with respect to approximation errors. In the frequency domain (FD) representation the approximation (3) yields an relative phase shift of

$$
\begin{gathered}
\Delta \varphi_{r e l}=-\boldsymbol{e}_{\boldsymbol{p}} \cdot \boldsymbol{e}_{\boldsymbol{q}} \cdot r_{n} \frac{1-\sqrt{1+r_{n}^{2}-2 \boldsymbol{e}_{\boldsymbol{p}} \boldsymbol{e}_{\boldsymbol{q}} r_{n}}}{1+\sqrt{1+r_{n}^{2}-2 \boldsymbol{e}_{\boldsymbol{p}} \boldsymbol{e}_{\boldsymbol{q}} r_{n}}}+ \\
+\frac{r_{n}^{2}}{1+\sqrt{1+r_{n}^{2}-2 \boldsymbol{e}_{\boldsymbol{p}} \boldsymbol{e}_{\boldsymbol{q}} r_{n}}} \leq \frac{r_{n}^{2}}{1+\sqrt{1+r_{n}^{2}}}
\end{gathered}
$$

with the normalized source distance $r_{n}=r_{q} / r_{p}$. Using this upper limit of the approximation error one can show that if the normalized source distance is less than $0.3\left(r_{n} \leq\right.$ $\left.0.3 ; r_{p} \geq 3 r_{q}\right)$ the relative phase error will be less than $5 \%$. 
The amplitude error resulting from the approximation (4) can be calculated by

$$
\Delta_{r e l}=\frac{\lambda}{r_{p} 2 \pi\left(1+r_{n}^{2}\right)}+\frac{1-\sqrt{1+r_{n}^{2}}}{\sqrt{1+r_{n}^{2}}}
$$

Using the determined ratio between the observation distance and the wavelength the amplitude error will be smaller than $5 \%$ if $r_{n} \leq 0.3\left(r_{p} \geq 3 r_{q}\right)$. Please note that in case $r_{n} \leq 0.1$ and $r_{p} \geq 12 \lambda$ the approximation error is less than $1 \%$.

\section{Near field dispersion}

After the well known frequency domain limits of the far field approximations have been expressed, the behavior of the fast transient radiation will be investigated. Assuming $\partial E_{a} / \partial t \gg$ $E_{a} c / r_{p}$ the time domain (TD) expression of (1) is:

$$
\begin{gathered}
\boldsymbol{E}_{\boldsymbol{S}}\left(\boldsymbol{r}_{\boldsymbol{p}}, t\right)=\frac{1}{2 \pi c r_{p}} \\
\int_{A_{a}}\left[\left(\boldsymbol{e}_{z} \times \frac{\partial}{\partial t} \boldsymbol{E}_{\boldsymbol{a}}\left(t+\frac{\boldsymbol{e}_{\boldsymbol{p}} \boldsymbol{r}_{\boldsymbol{q}}}{c}-\frac{r_{p}}{c}\right)\right) \times \boldsymbol{e}_{\boldsymbol{p}}\right] \\
\delta\left(t+\frac{\boldsymbol{e}_{\boldsymbol{p}} \boldsymbol{r}_{\boldsymbol{q}}}{c} \frac{1-\sqrt{1+r_{n}^{2}-2 \boldsymbol{e}_{\boldsymbol{p}} \boldsymbol{e}_{\boldsymbol{q}} r_{n}}}{1+\sqrt{1+r_{n}^{2}-2 \boldsymbol{e}_{\boldsymbol{p}} \boldsymbol{e}_{\boldsymbol{q}} r_{n}}}-\right. \\
\left.-\frac{r_{q}}{c} \frac{r_{n}}{1+\sqrt{1+r_{n}^{2}-2 \boldsymbol{e}_{\boldsymbol{p}} \boldsymbol{e}_{\boldsymbol{q}} r_{n}}}\right) d A_{a}
\end{gathered}
$$

Hereby $*$ describes the convolution with respect to time and $\delta()$ the Dirac function. The convolution with the delayed Dirac function leads to a larger pulse width in the near and intermediate field. This near field dispersion can be ignored if the delay

$\Delta t \leq \frac{r_{q}}{c} \frac{r_{n}}{1+\sqrt{1+r_{n}^{2}}}$

between the shortest ray and a ray from an arbitrary point on the aperture is short compared to the rise time of the radiated signal. Using the frequency domain far field definition we get a delay of $\Delta t=0.15 r_{q} / c$. Particularly for fast transient signals and large aperture cross sections this time delay is not acceptable. For a distance independent pulse shape of transient radiation it is necessary that $\Delta t$ is small compared with the rise time of the radiated signal. For practical applications the rise time should be 10 times larger than the longest time delay $\left(\tau_{r} \geq 6 \Delta t\right)$. Based on this the transient far field is given by

$r_{p} \geq \frac{3 \cdot r_{q}^{2}}{c \cdot \tau_{r}}=\frac{r_{q}}{c} \cdot \frac{3 \cdot r_{q}}{\tau_{r}}$

Unlike the frequency domain equation in this time domain equation the time $\left(r_{p} / c\right)$ which the wave needs to cross the aperture is weighted with the rise time as a measure of the shortest change in the shape of the signal. By comparing the phase shift with the phase of one oscillation of a cw-signal, one can get a similar relation in the frequency domain.

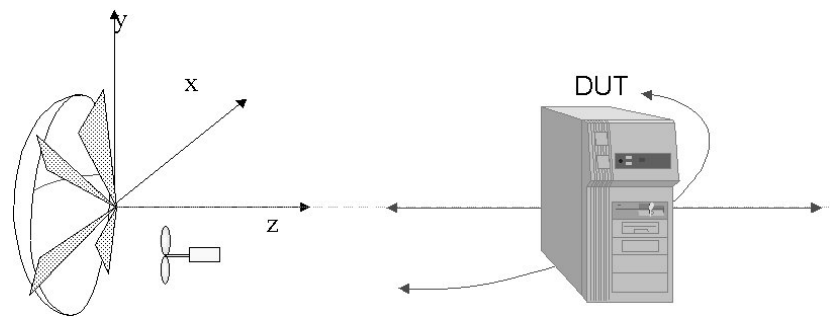

Fig. 1. Principle of the test setup for transient susceptibility investigations.

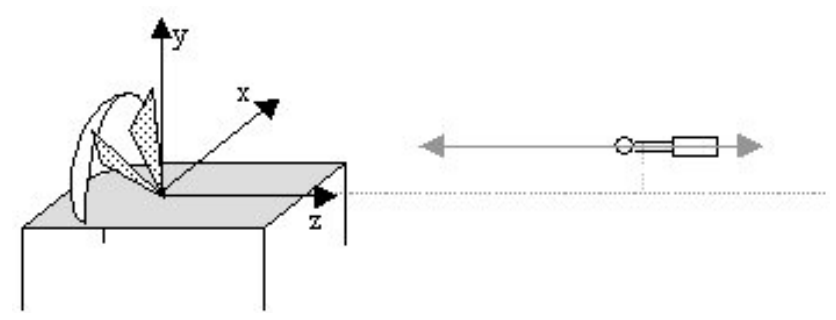

Fig. 2. Principle of the measurement setup.

\section{Examples}

In susceptibility test the impact of the angle between the main beam of the antenna and the direction of the EUT is generally taken into consideration, whereas usually the near field dispersion is ignored. The disregard of the near field effects is based on the one hand on the use of frequency domain rules and on the other hand on inadequate calibration. In order to demonstrate the near field dispersion measurements with an UWB-Demonstrator HIRA II were performed. The HIRA II is a half reflector type impulse radiating antenna consisting of a reflector with a diameter of $1.8 \mathrm{~m}$ on a $2 \mathrm{~m} \times 2 \mathrm{~m}$ metallic plate. The antenna was fed by a voltage pulse of double exponential shape with a rise time of $\tau_{r}=100 \mathrm{ps}$. The vertical component of the electric field strength was calculated and measured at different distances from the aperture plane and with a vertical offset of $0.3 \mathrm{~m}$ to the level of the metallic plate (Fig. 2).

The calculated and measured response of the HIRA II is shown in Fig. $3\left(r_{p}=2 \mathrm{~m}\right)$ and Fig. $4\left(r_{p}=10 \mathrm{~m}\right)$. With respect to limitations of the measurement setup in both diagrams the agreement between the computed and measured signals is excellent. The near field dispersion can be noticed clearly by comparing both pictures. At $r_{p}=2 \mathrm{~m}$ the pulse shape is different from the expected gaussian shape and the pulse width is more than 5 times longer than the rise time of the source signal. Increasing the observation distance to $10 \mathrm{~m}$, the pulse width decreases to one third of the rise time of the feeding voltage signal and the pulse looks like a gaussian pulse as expected.

In Fig. 5 the pulse amplitude is depicted as a function of the observation distance. Due to the influence of the near field dispersion the pulse amplitude decreases proportional to the observation distance. In a superficial interpretation one 


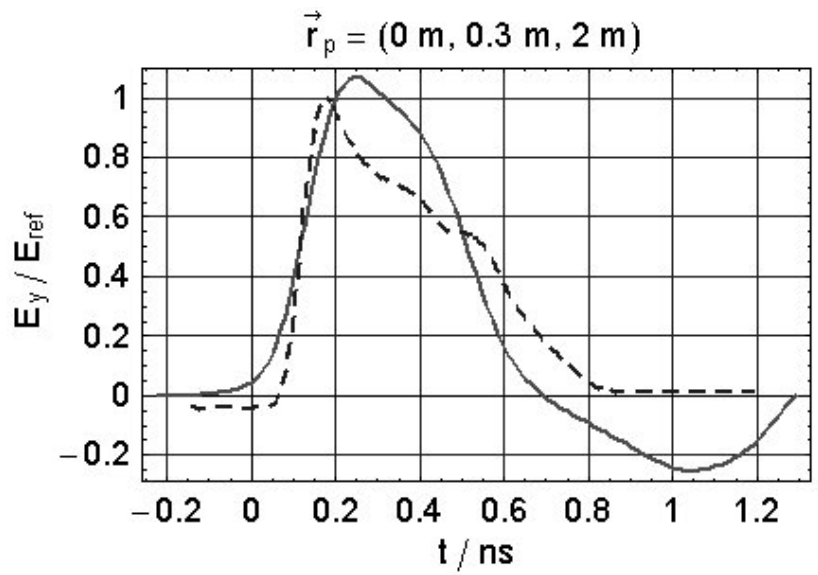

Fig. 3. Field pulse at $2 \mathrm{~m}$ (solid: measured; dashed: computed).

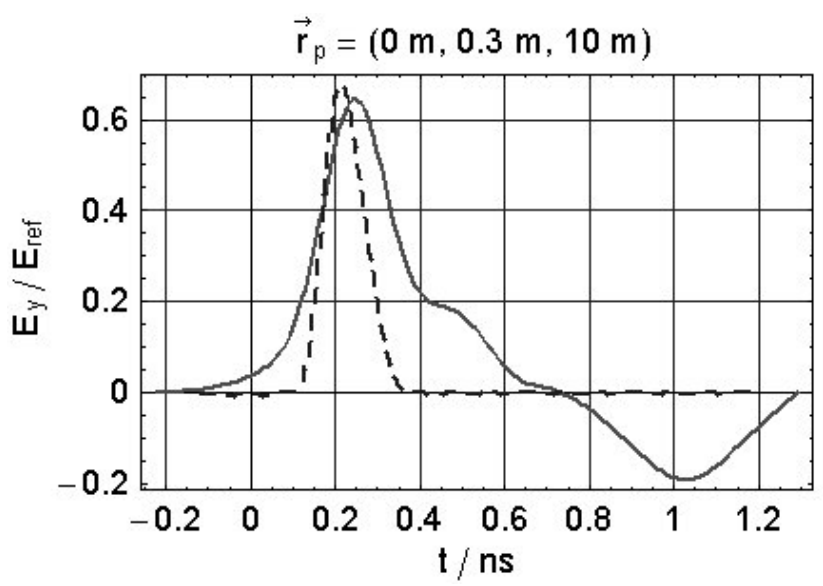

Fig. 4. Field pulse at $10 \mathrm{~m}$ (solid: measured; dashed: computed).

could use the shown curves to vary the strength of the electromagnetic field at the surface of an EUT by moving the EUT closer to or further away from the antenna. Unfortunately the pulse shape has to be considered as well as the amplitude because deviations of the shape cause significant changes of the coupling behavior. As a measure of such deviations the measured and the computed pulse widths are shown in Fig. 6 . A significant deviation between the measured and calculated pulse width can be observed, in contrast to the good agreement between the measured and calculated pulse amplitudes. The constant pulse width measured at distances $r_{p} \geq 7 \mathrm{~m}$ is caused by the bandwidth (or rise time) limitations of the measurement setup and does not indicate the transient far field. If the measurement were performed under far field conditions the rise time of the field pulse would have been comparable to the pulse width. Summarizing, the limited bandwidth of the measurement chain could lead to a misinterpretation of the calibration results.

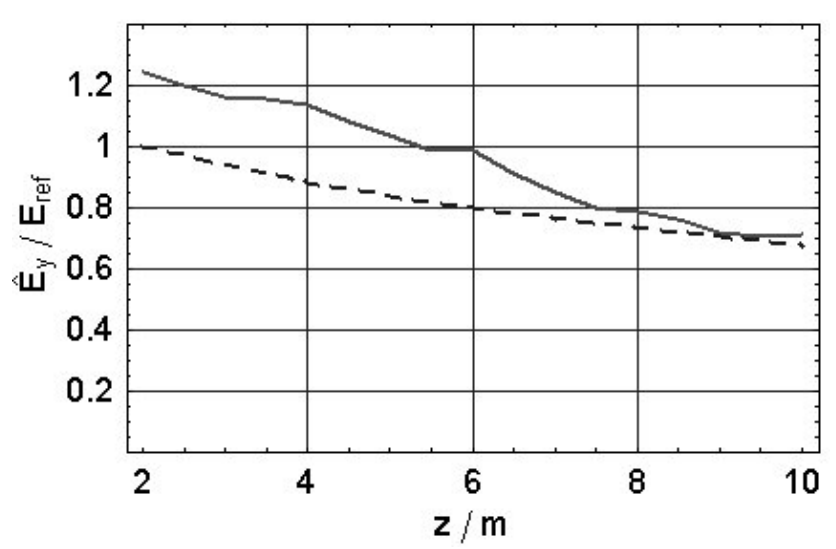

Fig. 5. Pulse amplitude (solid: measured; dashed: computed).

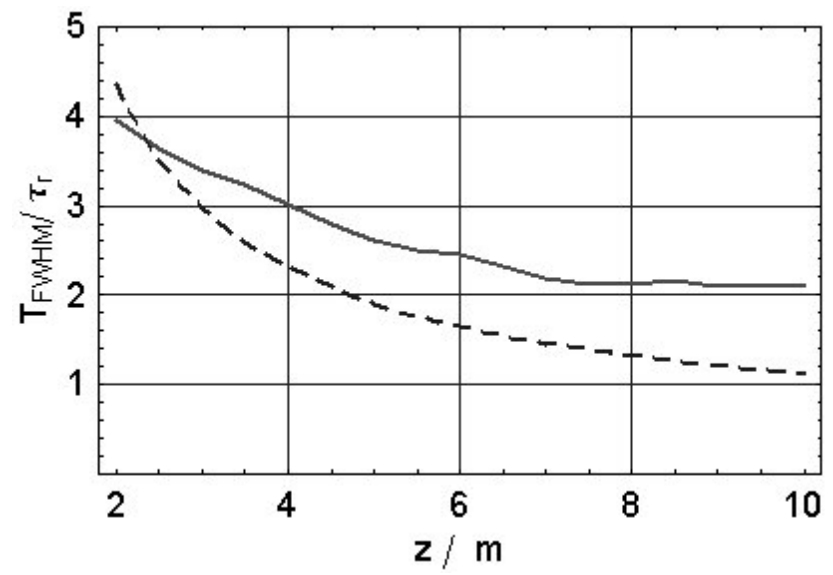

Fig. 6. Pulse width $\left(\tau_{r}=100 \mathrm{ps}\right)$ (solid: measured; dashed: computed).

\section{Peculiarities of transient susceptibility test}

The long distance where the transient far field begins $(<50 \mathrm{~m})$ is an essential problem for transient susceptibility measurements. On the one hand the change of the measurement distance can be used to vary the amplitude of the field pulse only under far field conditions. One the other hand usable distances $(10 \mathrm{~m}-30 \mathrm{~m})$ inside unechoic chambers are shorter than the far field distance. Only a few of the suitable open area test sides in Europe are permitted to generate the high field amplitudes which are necessary to generate several $\mathrm{kV} / \mathrm{m}$ in the far field. Based on simple frequency domain rules for the far field of an antenna and with regard to the test sides available, many transient susceptibility measurements are performed in the transient near field. Finally we would like to focus our interest on the impact of the near field dispersion on such susceptibility tests.

As an example for antenna systems used, we calculate the transfer function of the HIRA II at various distances. The 


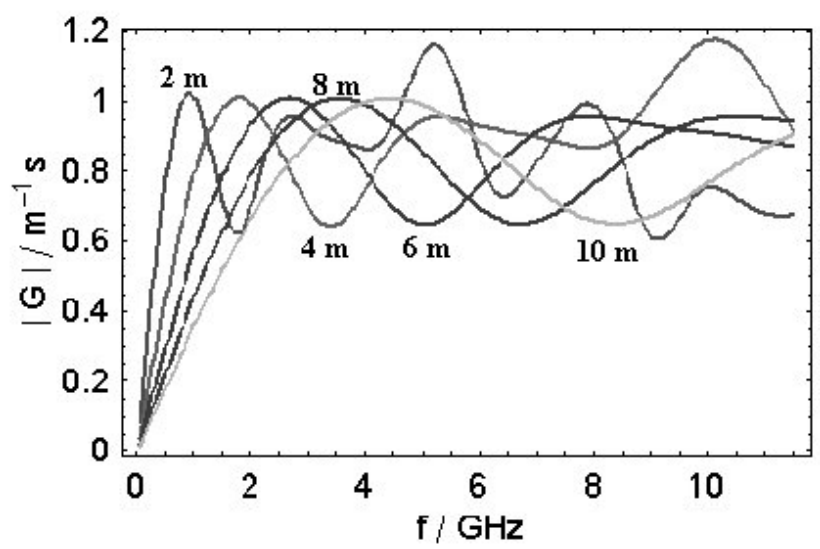

Fig. 7. Transfer function at various distances.

transfer functions between the feeding voltage signal and the field strength at observation points on the main beam at distances between $2 \mathrm{~m}$ and $10 \mathrm{~m}$ were calculated and are shown in Fig. 7. Up to $1 \mathrm{GHz}$ the transfer function increases proportionally to the frequency and decreases inversely proportional to the distance over the whole distance range shown. Above $3 \mathrm{GHz}$ all transfer function shown alternate around the value of 0.8. This non proportional behavior of the transfer function leads to a distortion of the field pulse. For susceptibility investigations it is necessary to assess this distortion of the radiated signal with regard to the spectrum of the feeding signal and the spectral susceptibility behavior of the EUT.

In contrast to the decrease of the pulse amplitude, the increase of the distance could raise the value of the transfer function at single frequencies. Particularly the magnitude at the $2 \mathrm{GHz}$ point of the transfer function of the HIRA II is equal at $2 \mathrm{~m}$ and $10 \mathrm{~m}$ distance. If one doubles the distance to $4 \mathrm{~m}$ the transfer function as well as the field spectrum magnitude (Fig. 8) at $2 \mathrm{GHz}$ will nearly be doubled whereas the pulse amplitude decreases to $80 \%$ (Fig. 5). In case the EUT is vulnerable (e.g. resonant) at $2 \mathrm{GHz}$ the change of the position from $2 \mathrm{~m}$ to $4 \mathrm{~m}$ can result in disruptions of the EUT. If this effect occurs it will be often assigned to the nonlinear behavior of components, because interaction models which assume far field conditions and only use the amplitude of the field pulse are not able to explain it. With regard to this unfavorable near field effects transient susceptibility measurements should be performed in the far field. In case the near field of an antenna must be used for a transient susceptibility test the following aspects should be taken into account:

1. The quality of the measurement chain limits the quality of the calibration procedure (e.g. beginning of the far field). The calibration method should take into account all parameters (amplitude, pulse width, rise time, pulse shape). For a correct calibration it is necessary that the inherent rise time of the measurement chain is ten times shorter than the expected rise time of the field signal.

2. During transient susceptibility test all parameters like pulse shape, amplitude, rise time and pulse width should

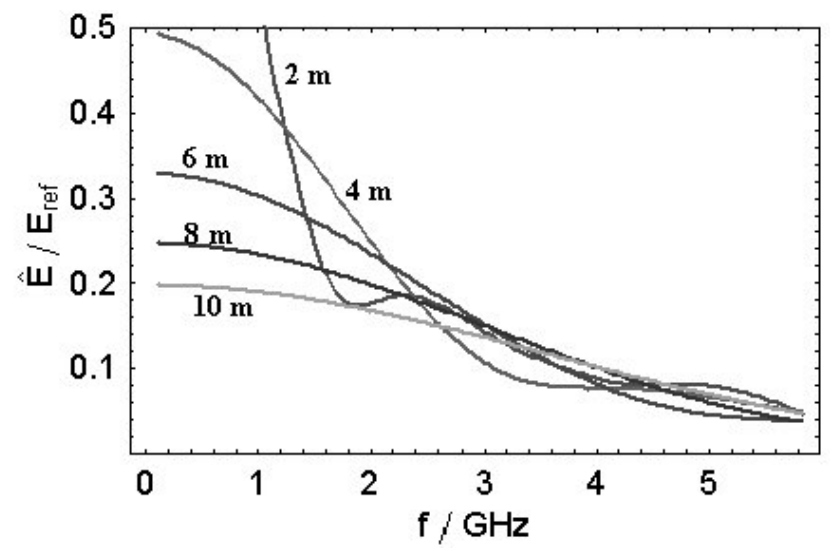

Fig. 8. Spectrum of a given field pulse at various distances.

be measured. Unwanted near field effects can only be avoided by an interpretation of the whole spectrum (magnitude and phase) of the incident field pulse.

3. In case the resonance of the EUT is known, the far field conditions can be assumed if the highest resonance frequency lies on the proportional part of the transfer function.

\section{Conclusion}

This paper investigates the effect of distance changes on the shape of the radiated field. Main aspects were near field effects as well as the prediction of the beginning of far field conditions of transient radiation. It is shown that the dimension of antenna systems and the wave propagation resulted in a special kind of dispersion. As a result the pulse shape of the radiated field signal is influenced by distance variations in the near field of the antenna. The importance of the near field dispersion for susceptibility investigations was demonstrated by theoretical considerations and measurements with a half pulse radiation antenna.

\section{References}

Balanis, C.: Antenna Theory, John Wiley, New York, 1997.

Sabath, F., Nitsch, D., Jung, M., and Weise, T.: Design and setup of a short pulse simulator for susceptibility investigations, Proceedings of the 13th IEEE International Pulsed Power Conference, Las Vegas (NV, USA), June 2001.

Sabath, F.: Near field dispersion of pulse radiation, Proceedings of the 27th URSI General Assembly, Maastricht (NL), August 2002.

Farr, E. and Sower, G.: Design Principles of Half Impulse Antennas, Sensor and Simulation Note 390, US Air Force Research Laboratory, Albuquerque (NM, USA), December 1995.

Farr, E. and Baum, C.: Extending the Definitions of Antenna Gain and Radiation Pattern Into the Time Domain, Sensor and Simulation Note 350, US Air Force Research Laboratory, Albuquerque (NM, USA), November 1992. 